Casos Clínicos

Arch. Esp. Urol., 59, 7 (732-736), 2006

\section{FRACTURA DE PENE CON ROTURA ASOCIADA DE URETRA.}

Roberto Llarena Ibarguren, Ainara Villafruela Mateos, lgor Azurmendi Arin, Jorge García Fernández y Carlos Pertusa Peña.

Servicio de Urología. Hospital de Cruces. Osakidetza. Bilbao. Vizcaya. España.

Resumen.- OBJETIVO: Presentamos dos casos de fractura de pene, 1 con fractura asociada de cuerpo esponjoso y sección completa de uretra y otro que sufrió la segunda fractura cavernosa 3 años después de la primera.

MÉTODOS: El primer paciente, de 32 años, sufrió la fractura del cuerpo cavernoso derecho durante el coito, 3 años después de haber sufrido una primera fractura de la que fue intervenido de urgencia. En este caso también fue intervenido de inmediato evidenciando la lesión y reparándola, con buenos resultados funcionales. El segundo paciente, de 29 años, sufrió la fractura transversal de ambos cuerpos cavernosos a nivel basal asociándose la sección completa de la uretra en una misma línea de fractura. Intervenido de urgencia se solucionaron todas las lesiones, con buenos resultados estéticos y funcionales.
Roberto Llarena lbarguren

Apartado de correos 20134

48080 Bilabao. Vizcaya. (España)

rllarena@euskalnet.net

Trabajo recibido: 12 de diciembre 2005
RESULTADOS: Dentro de la rareza que supone la lesión peneana traumática con fractura cavernosa, la asociación de lesión uretral supone una excepción, así como la posibilidad de sufrir una segunda fractura años después de la primera.

CONCLUSIONES: La fractura peneana supone una rara urgencia que debe ser reparada de forma inmediata evacuando el hematoma, suturando la lesión albugínea y solucionando la lesión uretral en los raros casos en que se asocie. Los resultados serán mejores, evitándose complicaciones a medio y largo plazo.

Palabras clave: Fractura de pene. Cuerpo cavernoso. Albugínea. Uretra.

Summary.- OBJECTIVE: We report two cases of penile fracture; one case with associated rupture of the corpus spongiosum and complete urethral section, and the other presenting a second cavernous fracture three years after a first episode.

METHODS: The first patient, 32 years old, suffered the fracture of the right corpus cavernosum during intercourse three years after a first fracture requiring surgical repair. This time he also underwent surgery immediately to find and repair the lesion, with good functional outcome. The second patient,29 years old, suffered a transverse fracture at the bases of both corpora cavernosa associated with complete section of the urethra. He underwent emergency surgery to repair all lesions with good esthetic and functional results.

RESULTS: Traumatic lesions of the penis with fracture of the corpora cavernosa are rare and the association with urethral rupture is exceptional, as well as it is the possibility of second fracture years after a first episode.

CONCLUSIONS: Penile fracture is a rare reason for consultation in the emergency setting, which should be repaired immediately with evacuation of the hematoma, closure of the albuginea's lesion and repair of the urethral lesions when they appear. Results are better, avoiding mid and long-term complications.

Keywords: Penile fracture. Corpus cavernosum. Albuginea. Urethra.

\section{INTRODUCCIÓN}

La fractura de pene se define como la rotura de 1 o de ambos cuerpos cavernosos asociada o no a lesión del cuerpo esponjoso, que puede estar acompañada de lesión uretral, y cuya causa más frecuente es un traumatismo directo y cerrado sobre el pene en erección. 
Es una lesión rara, que presenta una incidencia calculada de 1 caso por cada 175.000 ingresos hospitalarios (1). Es posible que algunas lesiones incluso ni se diagnostiquen por inasistencia del paciente a un centro hospitalario. La lesión uretral asociada a ella es aun más rara, ocurriendo en el $20-30 \%$ de los casos según las diferentes comunicaciones $(2,3,4,5,6,7)$, siendo más frecuente la lesión esponjosa sin afectación uretral propiamente dicha.

Fue descrita por primera vez en 1925 por Malis (8). La mayor parte de casos comunicados son únicos o todo lo más de dos casos, salvo los 9 publicados por Tejido (9). Seguramente la mayoría de los casos no se publican. Según Franco de Rose (10) hasta 2001 eran cerca de 400 los casos publicados. Aunque tenemos que tener en cuenta que en 2000, tan solo un autor, Zargooshi, publicó 172 casos, recogidos desde abril de 1990 hasta octubre de 1999 (11). El último caso presentado como video, que incluía lesión completa uretral, lo fue en Congreso Nacional de Urología de San Sebastián en mayo de 2005 (12).

No hemos encontrado en la literatura consultada ningún caso de fractura "recidivante" cavernosa, similar a la ocurrida en uno de nuestros casos.

\section{CASOS CLínICOS}

Caso 1. Varón de 32 años que acudió al Servicio de Urgencias por traumatismo peneano ocurrido durante el coito, 1 hora antes. Refería detumescencia inmediata, tras el ruido de un "chasquido" que coincidió con dolor intenso a nivel peneano. Tras esto apareció de forma inmediata aumento del tamaño peneano con deformidad a la izquierda, unido a aparición de hematoma subcutáneo, que terminaba a la altura de la raíz penea-

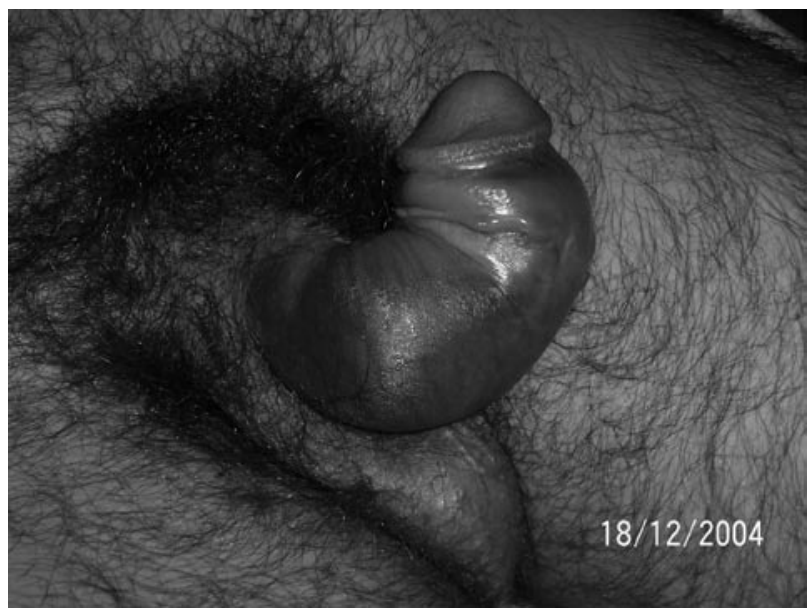

FIGURA 1. Hematoma de cubiertas con desviación peneana a la izda. na (Figura 1). Se da la casualidad que 3 años antes le ocurrió exactamente lo mismo, por lo que fue operado 24 horas después. En estos 3 años las erecciones y las relaciones sexuales fueron normales. Es intervenido de forma urgente, evidenciando fractura de $2 \mathrm{~cm}$. en cuerpo cavernoso derecho, a unos $2 \mathrm{~cm}$. del borde glandular, transversal y muy próxima al cuerpo esponjoso. No se evidenció uretrorragia ni lesión uretral. Se practicó sutura con material reabsorvible 00 en puntos sueltos. El resultado a posteriori fue excelente.

Caso 2. Varón de 29 años que acudió al Servicio de Urgencias por intensa uretrorragia tras traumatismo peneano durante la realización de coito. Refirió dolor súbito peneano seguido de detumescencia inmediata y deformidad peneana con angulación ventral desde la base peneana. La uretrorragia que refería como intensa había cesado. Durante la exploración se constató la imposibilidad al sondaje a los $10 \mathrm{~cm}$. del meato. Con el diagnóstico de fractura de pene y la sospecha de lesión uretral se procedió a intervención de urgencia. Se constató una lesión de la albugínea de $5 \mathrm{~cm}$. de longitud que interesaba a ambos cuerpos cavernosos a nivel de la base peneana, y que se acompañaba de sección uretral completa (Figura 2). Se procedió a sutura cavernosa con puntos sueltos de material reabsorvible 00 , y sutura uretral previa espatulación de los bordes, también con material reabsorvible 000. Los resultados tanto estéticos como funcionales miccionales han sido satisfactorios.

\section{DISCUSIÓN}

El pene en estado fláccido se encuentra protegido frente a diferentes traumatismos dada precisamente esa movilidad. La mayor parte de las fracturas peneanas en nuestra latitud ocurren durante maniobras coitales intempestivas al golpear el pene erecto tras su salida de vagi-

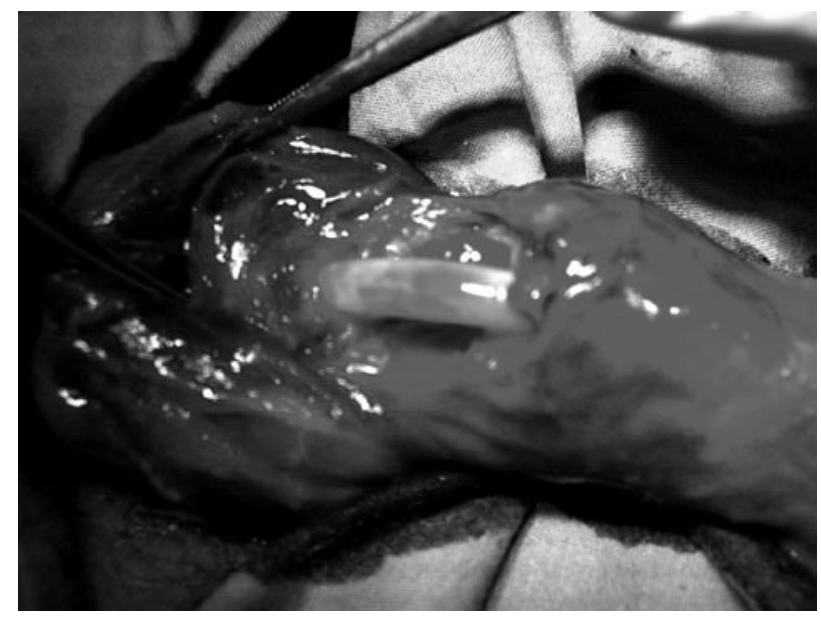

FIGURA 2. Línea de fractura transversal que incluye ambos cuerpos cavernosos y la uretra. 
na contra el periné o contra el pubis de la pareja, movimiento que incluso ha recibido distintas denominaciones como "faux pas de coit", "Texas trauma" o "bent nail síndrome (1). Normalmente ocurre cuando la mujer está situada encima del varón durante la relación sexual. Por el contrario en Asia, la fractura peneana puede tener lugar durante una serie de maniobras forzadas que pretenden detumescer el pene erecto, que también tienen denominación, taghaandan, tal como refieren Lorenzo (1) y Tejido (9). Gil (8) en su trabajo expone dos series diferentes de autores en las que los datos en cuanto a los mecanismos productores son contrapuestos ya que en una de ellas la manipulación inconsciente del pene, seguida de la caída al suelo durante la erección, o el rodar en la cama son las causas principales de rotura peneana, requiriendo todas ellas que el pene se encuentre en erección.

De los casos nacionales consultados, 3 de los 9 casos de Tejido (9) y el caso de Gil (8) no ocurrieron durante maniobras coitales.

Una explicación para esta debilidad de la albugínea que la haga más frágil durante la erección es la disminución de su grosor, sobre los $2 \mathrm{~mm}$ en reposo, hasta los $0^{\prime} 25-0^{\prime} 50 \mathrm{~mm}$, disminuyendo de esta manera la elasticidad mientras aumenta la presión intracavernosa $(13,14)$. Aún así, autores como Lema (7) recuerdan que el $3 \%$ de las fracturas peneanas ocurren con el pene fláccido, sin que en estos casos participen lógicamente estos mecanismos.

Según Franco (10) hasta en el $83 \%$ de los pacientes pueden demostrarse factores patológicos de la albugínea, como fibroesclerosis de la misma que la harían más predispuesta a la lesión.

Clínicamente y por todos conocido la fractura peneana coital produce dolor intenso y agudo, aunque no siempre, unido a la audición del clásico "chasquido", seguidos de la desaparición inmediata de la erección, y aparición posterior y progresiva de hematoma que hace aumentar tanto el diámetro como la longitud peneana. Posteriormente aparece la incurvación peneana conforme crece el hematoma hacia el lado contrario a la lesión albugínea. A esta forma peneana, deformada, incurvada, junto con el color cereza que adquiere debido al hematoma de cubiertas se le denomina "en berenjena" (11). En los casos de integridad de la fascia de Buck el hematoma se limita al pene adquiriendo una morfología en "manga", mientras que si está lesionada el hematoma difundirá por escroto, ingles, periné incluso hipogastrio conteniéndose por la fascia de Colles, pudiendo adquirir la clásica morfología en "mariposa" (1).

Autores como Lorenzo (1) refieren que en ocasiones y ante hematomas de pequeño tamaño puede palparse el defecto del cuerpo cavernoso, incluso percibirse el coágulo firme, fijo e indoloro adherido a la lesión albugínea, denominándose a este signo como del "rodete" (7).
Esta rotura suele ser unilateral, transversal, y del lado derecho $(9,13,14)$, siendo más frecuente que tenga lugar en el tercio proximal peneano hasta en el $90 \%$ de los casos (11), aunque para otros la fractura acostumbra a producirse en el tercio distal (15). Por ejemplo, de los 9 casos referidos por Tejido (9), en 8 la rotura fue derecha, y en 5 de ellos, proximal. En cuanto a la cara peneana, tampoco existe unanimidad, ya que mientras unos refieren más frecuente la fractura en la cara ventral (3), otros por el contrario, la refieren en la cara dorsolateral (13).

La fractura puede acompañarse de rotura uretral hasta en un $38 \%$ de las ocasiones, según las series $(2-7$, $11,12)$. En este caso es más frecuente que la fractura sea bilateral, y deberemos sospecharla siempre que el paciente refiera uretrorragia, aparición de sangre en el meato uretral, hematuria, dificultad en el sondaje o imposibilidad para la micción, aunque estas situaciones ocurran a veces por la compresión uretral del hematoma y no por rotura uretral. Algunos refieren que el hinchazón peneano coincidente con la micción nos debe hacer sospechar la lesión uretral (8). Las lesiones uretrales acostumbran a ser pequeñas y parciales por lo que muchas pudieran pasar desapercibidas (8).

Ante la sospecha de lesión uretral el cateterismo uretral deberá ser evitado por el riesgo de convertir una rotura uretral parcial en completa $(2,3,4,5,16)$. Incluso algunos proscriben el sondaje uretral aunque no existan datos ni signos sospechosos de afectación uretral (16). Ante la sospecha de lesión uretral debiera de realizarse para algunos, estudio uretrográfico miccional previa cistostomía suprapúbica $(1,7,14)$. El estudio uretral radiológico no está exento de falsos diagnósticos. Esta medida nos parece un tanto desproporcionada ya que la cirugía inmediata solucionará tanto la fractura albugínea como la uretral siendo necesario para ésta última el sondaje uretral.

Dentro de las pruebas complementarias puede realizarse ecografía peneana, prueba de mayor rendimiento por su fiabilidad, bajo coste y gran disponibilidad, en la que se puede apreciar la solución de continuidad de la albugínea, y si además la acompañamos de un estudio doppler estudiar la vascularización peneana de cara a realizar el diagnóstico diferencial $(7,13,15,17)$. El estudio sonográfico permite visualizar el hematoma, incluso en ocasiones la fractura cavernosa como un área hipoecoica que interrumpe la continuidad de la albugínea (14). Normalmente para interpretar estos signos hace falta mucha experiencia, que habitualmente y dada la escasa incidencia de estos trastornos es muy difícil de conseguir, por lo que la probabilidad tanto de falsos positivos como de negativos será alta (14).

La cavernosografía es una técnica invasiva, difícil de realizar de urgencia en la mayoría de los hospitales, fiable, aunque no exenta de complicaciones potenciales, como fibrosis cavernosa e infección $(1,7,13,14)$. 
Una vez introducidos en un cuerpo cavernoso unos 50 cc de material de contraste es necesario esperar unos 10 minutos ya que a veces el coágulo puede retrasar la extravasación. La única contraindicación sería la hipersensibilidad del paciente a los contrastes yodados. Su principal utilidad sería ayudar al cirujano a planificar la intervención, localizando la ruptura, y detectando otras pequeñas posibles roturas que no se hayan manifestado clínicamente e incluso puede llegar a excluir comunicación con la uretra (9). Aparte de que puede retrasar la intervención quirúrgica, no la consideramos fundamental salvo en casos de seria duda diagnóstica y solo para demostrar la completa integridad de la albugínea. Como ejemplo, Zargooshi (1 1) no la practica en ninguno de sus 172 casos, por no necesitarla como medio diagnóstico y para evitar posibles priapismos..

La resonancia nuclear magnética (RMN) es la prueba de mayor fiabilidad pero de coste muy elevado y baja disponibilidad en nuestro medio (18). En ella la rotura albugínea se observa como una interrupción en la señal de baja intensidad característica de esta estructura en fase T2 (18). En teoría la RMN puede localizar el lugar exacto de la fractura albugínea pudiendo realizar una menor incisión para su reparación, evitando la denudación completa peneana.

Algunos autores han descrito la posibilidad de inyectar intracavernosamente PGE 1 para valorar el crecimiento del hematoma y desviación del pene secundaria en los casos de duda diagnóstica, ya que en los casos que presentan rotura estos signos se acentuarán (19). Por último, algunos autores aconsejan que cuando en la exploración quirúrgica no encuentran la lesión, realizan una erección artificial mediante la inyección intracavernosa de suero fisiológico para poner de manifiesto la rotura $(13,20)$. Aunque no hemos tenido la necesidad nos parece una opción interesante.

El diagnóstico diferencial de esta patología, tal como refieren la mayoría de los autores, debe establecerse esencialmente con la rotura de la vena dorsal profunda del pene, producida habitualmente por un mecanismo traumático idéntico y que se manifiesta clínicamente de forma similar $(1,2,4,5,8,10,13,21)$. No existen en la literatura casos de fractura albugínea concomitante con lesión de las venas peneanas tanto la dorsal como la profunda (13).

Para el tratamiento en la literatura consultada encontramos dos alternativas (22). Hasta la década de los 70 el tratamiento conservador consistente en vendaje compresivo, sonda uretral o cistostomia ante la dificultad para el sondaje, o sospecha de lesión uretral, frío local, reposo, antibióticos, elevación del pene, antiinflamatorios o enzimas proteoliticas y medicación para evitar erecciones como antiandrógenos, diazepam, etc. Esta alternativa presenta una tasa de complicaciones entre el 10 al $80 \%$, según los autores $(1,7-9,13,14,15,17$, $20,22)$. Entre estas complicaciones podemos encontrar una masa o divertículo pulsátil por debilidad albugínea, erecciones dolorosas, sobreinfección del hemato$\mathrm{ma}$, incurvación peneana residual, estenosis de uretra, disfunción eréctil, placa fibrótica, fístula uretral tanto cavernosa como cutánea (23), fístulas arterio-venosas, mayor estancia hospitalaria, etc. En la actualidad la mayoría de los autores prefieren el tratamiento quirúrgico, realizando la sutura de la fractura de la albugínea de forma inmediata. Este tipo de tratamiento presenta una menor tasa de complicaciones, menor estancia hospitalaria, menor probabilidad de deformidades peneanas y una recuperación de las relaciones sexuales más rápida $(1,12-14)$.

El abordaje quirúrgico más utilizado es la incisión circunferencial subcoronal continuada con la denudación del pene hasta su base. Esta incisión nos permite un buen abordaje de los cuerpos cavernosos y la uretra, permitiéndonos una correcta exploración de la lesión, evacuación del hematoma y reparación de la albugínea. Otros abordajes posibles son la incisión corporal longitudinal sobre el hematoma y la incisión inguinoescrotal (24). Con el abordaje longitudinal, podemos tener problemas a la hora de evacuar el hematoma, de inspeccionar la uretra y el otro cuerpo cavernoso. Por eso esta incisión sólo se recomienda en el caso de lesiones pequeñas $(7,9,22)$. Los partidarios del abordaje inguino-escrotal refieren que esta incisión es mejor que las otras dos porque maneja tejidos no edematizados (24).

Nosotros al igual que otros autores consultados pensamos que a pesar de manejar tejidos sin alteraciones, esta incisión puede presentar problemas en la evacuación del hematoma y en la correcta exploración del pene en su totalidad anatómica. La sutura de la túnica albugínea la realizan la mayoría de los autores con material reabsorbible de 00-000 con sutura discontinua $(1-6,8-9,12)$. Curiosamente Zargooshi (11) realiza la sutura con hilo irreabsorvible, aunque parece aceptado por la mayoría que esta sutura puede provocar molestias incómodas al paciente ya que los nudos se pueden palpar quedando evidentes bajo la piel peneana. Están descritas técnicas reparativas de grandes defectos de la albugínea mediante parches (11). En el postoperatorio se debe colocar un vendaje compresivo, suficiente para evitar colecciones, evitando así el empleo de drenajes y sonda vesical durante 24-48 horas hasta que la regresión del edema permita una micción cómoda al paciente, pudiéndose añadir medidas para evitar erecciones $(1,5,6,9)$. Nosotros no vemos, al igual que otros autores, la necesidad de dejar drenajes si no ha habido ninguna complicación durante la cirugía.

En el caso de sospecha de lesión uretral, la mayoría de los autores recomiendan la realización de una cistostomía suprapúbica, lo que evita la extravasación de orina a la zona quirúrgica $(3-6,11,12)$, otros $(9,11,12)$ entre los que nos encontramos, preferimos la exploración y resolución del daño uretral concomitante a la cirugía re- 
parativa. Los resultados a largo plazo, en cuanto a aparición de estenosis, son similares a los obtenidos con la reparación inmediata del daño uretral (9). Suponemos que la actitud abstencionista en cuanto a la lesión uretral se referirá a las lesiones parciales uretrales, porque lo que parece claro es que ante la disrupción completa la cirugía inmediata será imperativa. Tanto en un caso como en otro, la sutura se realizará sobre sonda uretral, con puntos sueltos reabsorvibles, debiendo espatular los bordes cuando la fractura ha sido completa. Deberá mantenerse catéter uretral al menos 3 semanas.

\section{CONCLUSIONES}

La fractura de los cuerpos cavernosos es una entidad poco frecuente, relacionada con la actividad coital en nuestra latitud. Para su diagnóstico suele ser suficiente con la anamnesis y la exploración física, clásica y típica, aunque disponemos de diversas pruebas complementarias que nos pueden ayudar. De éstas, la ecografía es la de mayor rendimiento, por su sencillez y economía, aunque no está exenta de falsos negativos. Actualmente el tratamiento quirúrgico urgente es el preferido por la gran mayoría de los urólogos, dado el menor número de complicaciones a largo plazo que presentan los penes intervenidos de inmediato. La lesión uretral concomitante, en ocasiones completa, aunque rara, es posible, por lo que está indicada una correcta valoración esponjosouretral en todos los casos.

\section{BIBLIOGRAFIA y LECTURAS RECOMENDADAS (*lectura de interés $y^{* *}$ lectura fundamental)}

1. LORENZO, J.G.; SEGURA, M.; SALINAS, M. y cols.: "Rotura de cuerpos cavernosos: Evaluación y manejo terapéutico". Actas Urol. Esp., 22: 635, 1999.

2. WALTON, J.K.: "Fracture of the penis with laceration of the urethra". Br. J. Urol., 51: 308, 1979.

3. CUMMING, J.; JENKINS, J.D.: "Fracture of the corpora cavernosa and urethral rupture during sexual intercourse". Br. J. Urol., 67: 327, 1991.

4. KOWALCZYK, J.; ATHENS, A.; GRIMALDI, A.: "Penile fracture: an unusual presentation with lacerations of bilateral corpora cavernosa and partial disruption of the urethra". Urology, 44: 599, 1994.

5. RUBIO, J.; SÁNCHEZ, F.; SEGURA, A. y cols.: "Tratamiento quirúrgico de la fractura de cuerpos cavernosos y uretra: revisión de la literatura a propósito de dos casos”. Arch. Esp. Urol., 49: 499, 1996.

6. GROSS, M.; ARNOLD, T.L.; PETERS, P. y cols.: "Fracture of de penis with associated laceration of the urethra". J. Urol., 117: 725, 1997.
7. LEMA, J.; GRILLE, M.; BLANCO, M. y cols.: "Fractura de pene con afectación de cuerpos cavernosos y uretra". Actas Urol. Esp., 23: 900, 1999.

*8. GIL, P.; BORQUE, A.; VALDIVIA, P. y cols.: "Fractura de pene. Aportación de un caso". Actas Urol. Esp., 24: 272, 2000.

**9. TEJIDO, A.; MARTÍN, M.P; VILLACAMPA, F. y cols.: "Tratamiento quirúrgico de la fractura de pene. Nuestra experiencia". Actas Urol. Esp., 23: 784, 1999.

**10. FRANCO DE ROSE, A.; GIGLIO, M.; CARMIGNANI, G.: "Traumatic rupture of the corpora cavernosa: new physiopathologic acquisitions". Urology, 57: 319, 2001.

**11. ZARGOOSHI, J.: "Penile fracture in Kermanshah, Iran: report of 172 cases". J. Urol., 164: 364, 2000.

12. MORENO, J.; FERNÁNDEZ, L.; GORDO, E. y cols.: "Fractura de pene con rotura de uretra después de una relación sexual". Video presentado al LXX Congreso Nacional de Urología, San Sebastián, 4-7 de junio de 2005.

**13. SERRANO, A.; GOLBANO, J.M.; GONZÁLEZPERAMATO, P. y cols.: "Fractura de pene: evaluación diagnóstica y actitudes terapéuticas. Revisión de la literatura". Arch. Esp. Urol., 54: 803, 2001.

14. RODRÍGUEZ, A.; GONZÁLEZ, A.; CESPÓN, E. y cols.: "Fractura de pene. Aportación de un caso". Actas Urol. Esp., 25: 765, 2001.

15. MARIÑO, J.J.; SENOVILLA, J.; MATEOS, J. y cols.: "Fractura de pene. Revisión de la literatura y presentación de dos casos". Actas Urol. Esp., 24: 767, 2000.

16. EL-SHERIF, A.E.; DAULEH, M.; ALLOWNEH, N. y cols.: "Management of fracture of the penis in Qatar". Br. J. Urol., 68: 622, 1991.

17. MARTÍNEZ, E.; ARNAIZ, F.; PÉREZ, J.A. y cols.: "Fractura de pene: dos nuevos casos. Revisión de la literatura. Utilidad de la ecografía”. Arch. Esp. Urol., 50: 1099, 1997.

18. FEDEL, M.; VENZ, S.; ANDREESSEN, R. y cols.: "The value of magnetic resonance imaging in the diagnosis of suspected penile fracture". J. Urol., 155: 1924, 1996.

19. RODRÍGUEZ DE LEDESMA, J.M.; FERRERO, R.; FONTANA, L.O. y cols: "Utilidad de la prostaglandina E1 en el diagnóstico de la fractura de cuerpos cavernosos. A propósito de un caso". Arch. Esp. Urol., 50: 302, 1997.

*20. MYDLO, J.H.: "Surgeon experience with penile fracture". J. Urol., 166: 526, 2001.

21. NICELY, E.R.; COSTABILE, R.A.; MOUL, J.W.: "Rupture of the deep dorsal vein of the penis during sexual intercourse". J. Urol., 147: 150, 1992.

*22. HERRANZ, L.M.; AVELLANO, R.; JIMÉNEZ, M. y cols.: "Fractura de pene". Arch. Esp. Urol., 56: 1079, 2003.

23. HARGREAVES, D.G.; PLAIL, R.O.: "Fracture of the penis causing a corporo-urethral fistula". Br. J. Urol., 73: 97, 1994.

24. SEFTEL, A.D.; HAAS, C.A.; VAFA-BROWN, S.L.: "Inguinal scrotal incision for penile fracture". J. Urol., 159: 182, 1998. 